\title{
Erratum: Strong Monogamy Conjecture for Multiqubit Entanglement: The Four-Qubit Case [Phys. Rev. Lett. 113, 110501 (2014)]
}

\author{
Bartosz Regula, Sara Di Martino, Soojoon Lee, and Gerardo Adesso \\ (Received 15 January 2016; published 29 January 2016)
}

DOI: 10.1103/PhysRevLett.116.049902

The value of the lower bound to the reduced three-tangles $\tau^{(3)}$ in the second column of Table I for the representatives of class-2 states $\left|G_{a b c}^{2}\right\rangle$ was reported incorrectly in the Letter, as pointed out in [1]. The correct value should read

$$
\tau_{q_{i}\left|q_{j}\right| q_{k}}^{(3)} \leq \frac{4|c| \sqrt{\left(a^{2}-b^{2}\right)\left(a^{* 2}-b^{* 2}\right)}}{\left(|a|^{2}+|b|^{2}+2|c|^{2}+1\right)^{2}} .
$$

A corrected version of Fig. 2(a) of the original Letter has been plotted here as Fig. 1.

Additionally, the value of the lower bound to the reduced three-tangles $\tau^{(3)}$ for the representatives of class-7 states $\left|G^{7}\right\rangle$ in the same table was also reported incorrectly in the Letter. The correct value should read

$$
\tau_{q_{1}\left|q_{j}\right| q_{k}}^{(3)} \leq \frac{1}{4}, \quad \tau_{q_{2}\left|q_{3}\right| q_{4}}^{(3)}=0
$$

The errors have no effect on the conclusions of the Letter or on any other calculations therein.

The authors would like to thank A. Osterloh for bringing the error in class-2 states to their attention.

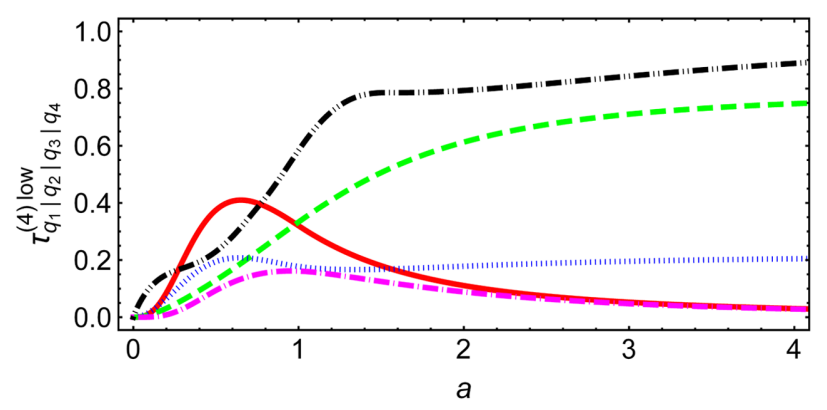

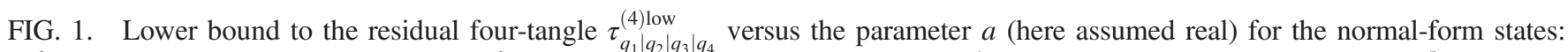
$\left|G_{a b c}^{2}\right\rangle$ with $b=c=a$ (red solid line), $\left|G_{a b}^{3}\right\rangle$ with $b=a / 4$ (green dashed line), $\left|G_{a b}^{4}\right\rangle$ with $b=a / 2$ (blue dotted line), $\left|G_{a}^{5}\right\rangle$ (magenta dot-dashed line), $\left|G_{a}^{6}\right\rangle$ (black dot-dot-dashed line). The residuals stay non-negative for general choices of the parameters $a, b, c$.

[1] A. Osterloh, The nine ways of four qubit entanglement and their threetangle, arXiv:1512.02468. 